\title{
NGR-DAU conjugates, a favorable tumor-homing motif with potential dual-targeting
}

Andrea Angelo Pierluigi Tripodi ${ }^{1}$, Szilard Toth $^{2}$, Ivan Randelovic ${ }^{3}$, Jozsef Tovari ${ }^{3}$, Gábor Mezó ${ }^{1}$

\author{
${ }^{1}$ MTA-ELTE Research Group of Peptide Chemistry, Hungary \\ ${ }^{2}$ Institute of Enzimology, Research Center for Natural Sciences, Hungarian Academy of Sciences, Hungary \\ ${ }^{3}$ National Institute of Oncology, Dept. of Experimental Pharmacology, Hungary
}

https://doi.org/10.17952/35EPS.2018.297

\section{Introduction}

At present, cancer is one of the major health-related issues world-wide. Strategies to target and defeat tumor are limited due to the access to tumor, side effects and absence of selectivity to the tumor tissue. Small molecule drug conjugates containing the Asn-Gly-Arg (NGR) motif can act as tumor-homing compounds that are recognized by Aminopeptidase N (APN/CD13). CD13 is receiving an accurate attention due to its role in the progression of tumor vasculo-genesis and identifying it as a critical regulator of angiogenesis, generally expressed on the monolayer cell surface of malignant cells $[1,2]$. As established in the literature the AsnGly-Arg sequence is inclined to Asn deamidation bearing the formation of a five-membered succinimide ring intermediate, that under fast hydrolytic conditions generates isoDGR derivative which can bind to integrins [3-6]. According to literature data, one of the most stable and tumor selective cyclic NGR-peptides is c[KNGRE]- $\mathrm{NH}_{2}$, in which the $\alpha$-amino group of the $\mathrm{N}$-terminal Lys is coupled to the $\gamma$-carboxyl group of the glutamic acid residue (head-to-side chain cycle) [7]. The preparation of a c[KNGRE] conjugate require a sophisticated synthetic route, for this reason the main goal of the present study was to investigate whether the exchange of the lysine in the cycle has any influence on the chemo-stability, selectivity and antitumor activity of the conjugates.

\section{Results and Discussion}

NGR cyclic peptides were prepared by SPPS on a Rink-Amide MBHA Resin, using Fmoc/tBu strategy. Daunomycin (Dau) as an anticancer agent was conjugated to an aminooxyacetic acid linker (oxime linkage) connected through an enzyme labile spacer (GFLG) via oxime linkage allowing an efficient drug release of active metabolite in lysosomes of cancer cells. We recently described that the Dau=Aoa-GFLGK(c[KNGRE]-GG-)$\mathrm{NH}_{2}$ conjugate $(\mathrm{K})$ has a significant anti-tumor activity against both CD13 positive HT-1080 human fibrosarcoma and CD13 negative but integrin positive HT-29 human colon adenocarcinoma cells [8]. However, we have confirmed that the free $\varepsilon$-amino group of Lys in the cycle is irrelevant for the biological activity. Therefore, we developed novel cyclic NGR peptide - daunomycin conjugates in which Lys was replaced by different amino acids [9]. From these investigations the Nle conjugate (3) as the most efficient compound was selected for further studies. The cytostatic and cytotoxic effect of the novel cyclic NGR peptide Dau conjugates were evaluated in vitro on HT-1080 (human fibrosarcoma), HT-29 (human colon adenocarcinoma) and KS (Kaposi's sarcoma) cell lines (Table 2). The stability in cell culture medium (Table 1) and the lysosomal degradation in presence of rat liver homogenate were determined by LC-MS [9]. 


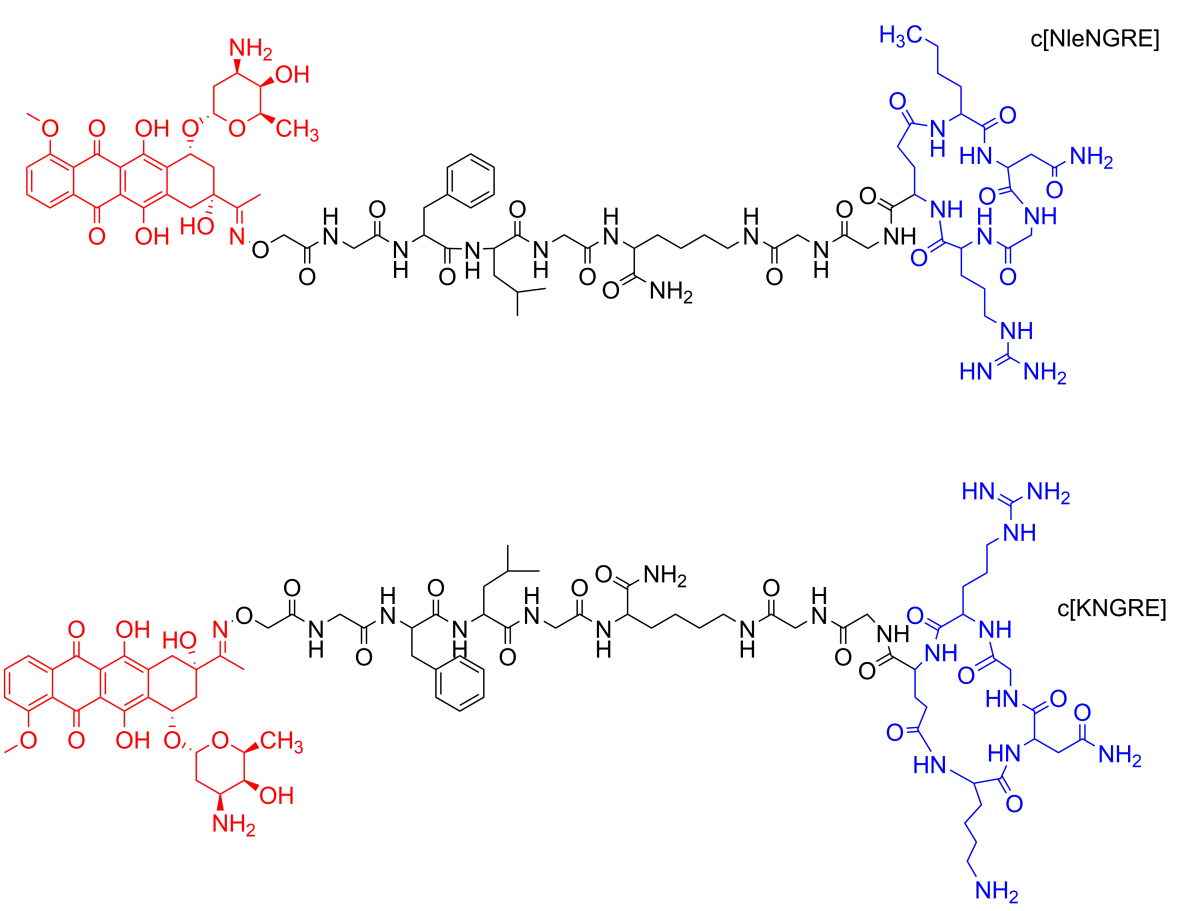

Figure 1

Table 1: Chemo-stability of cyclic NGR peptide - daunomycin conjugates

\begin{tabular}{|c|c|c|c|c|c|c|c|}
\hline \multicolumn{7}{|c|}{ Ratio of Asn-/Asp-/isoAsp-derivatives (DMEM CM, 37 ${ }^{\circ}$ C) } \\
\hline \multirow{2}{*}{ Code } & $\begin{array}{c}\text { AAA } \\
\text { replacement }\end{array}$ & \multicolumn{3}{|c|}{$\mathbf{6}$ h } & \multicolumn{3}{c|}{7} \\
\hline
\end{tabular}

The main cleavage site of the conjugates in lysosomes could be detected between Gly-Phe within the enzyme labile spacer resulting in the smallest active Dau containing metabolite Dau=Aoa-Gly-OH [9].

Detection of CD13 expression on tumorcell lines.

The surface expression of CD13 on KS (Kaposi's sarcoma) and on HT-1080 (human fibrosarcoma) was determined by immunocytochemistry with a FITC-conjugated anti-CD13 monoclonal murine antibody (OKM13). No fluorescence was detected in case of HT-29 (human colon adenocarcinoma) CD13 negative.

Table 2: In vitro cytostatic and cytotoxic effect IC50 ( $\mu M)$

\begin{tabular}{|l|l|c|c|c|c|c|c|}
\hline & & $\begin{array}{c}\text { HT1080 } \\
\mathbf{C D 1 3}+ \\
\mathbf{6 h}\end{array}$ & $\begin{array}{c}\mathbf{H T}-29 \\
\mathbf{C D 1 3}- \\
\mathbf{6 h}\end{array}$ & $\begin{array}{c}\text { KS } \\
\mathbf{C D 1 3}+ \\
\mathbf{6 h}\end{array}$ & $\begin{array}{c}\text { HT1080 } \\
\mathbf{C D 1 3}+ \\
\mathbf{7 2 h}\end{array}$ & $\begin{array}{c}\text { HT-29 } \\
\mathbf{C D 1 3}- \\
\mathbf{7 2 h}\end{array}$ & $\begin{array}{c}\text { KS } \\
\mathbf{C D 1 3} \\
\mathbf{7 2 h}\end{array}$ \\
\hline $\mathbf{3}$ & Dau=Aoa-GFLGK(c[NleNGRE]-GG-)-NH2 & 2.8 & 6.4 & 3.6 & 2.4 & 1.8 & 0.3 \\
\hline K & Dau=Aoa-GFLGK(c[KNGRE]-GG-)-NH2 & 12.4 & 9.9 & 7.2 & 5.7 & 16.5 & 2.4 \\
\hline Dau & Daunomycin & 0.004 & 0.03 & 0.04 & 0.002 & 0.001 & 0.001 \\
\hline
\end{tabular}


In this experiment we measured the cytostatic effect $(6 \mathrm{~h}$ treatment and further $66 \mathrm{~h}$ incubation after washing out the compounds)and the cytotoxic effect $(72 \mathrm{~h}$ treatment), compounds were dissolved in serum containing (FBS+) RPMI 1640 medium. Non-treated cells in both conditions were used as controls.

Cell Uptake studies

As daunomycin present fluorescent properties, internalization of both peptides was also detected by flow cytometry.

KS (6h)

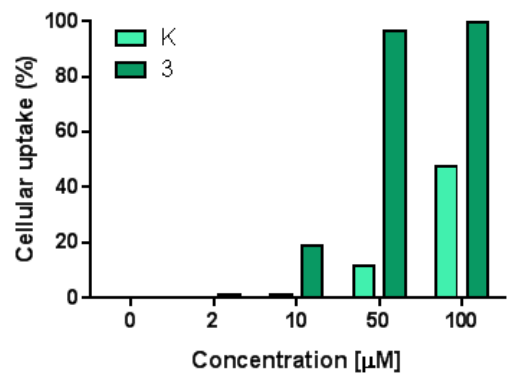

HT-29 (6h)

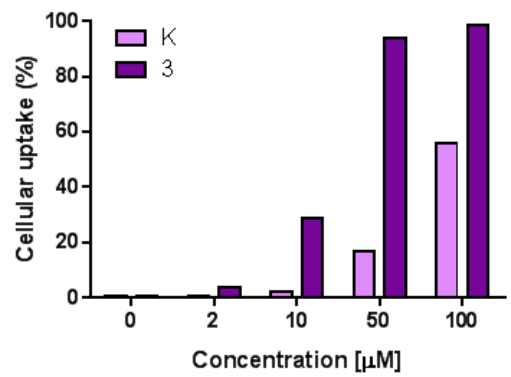

Figure 2

From this study we showed that the changes decrease the chemo-stability of the cyclic NGR moiety, resulting in the formation of isoAsp-derivatives in higher amount. Among the new cyclic NGR peptide - daunomycin conjugates the most effective compound was Dau=Aoa-GFLGK(c[Nle-NGRE]-GG)-NH2. This conjugate (3) was especially active on Kaposi's sarcoma cell which highly expresses for CD13.

\section{Acknowledgements}

This project had received fundings from the European Union's Horizon 2020 research and Innovation program under the Marie Sklodowska-Curie grant agreement No 642004, and from the National Research, Development and Innovation Office (NKFIH K119552), Hungary.

\section{References}

[1] Curnis, F.; Cancer Res 2002, 62, 867-74.

[2] Corti A.; Blood. 2008 Oct 1;112(7):2628-35.

[3] Meinwald, Y. C.; et al. Int. J. Pept. Protein Res. 1986, 28, 79-84.

[4] Kirikoshi, R.; Manabe, N.; Takahashi, O. Int. J. Mol. Sci. 2017, 18, No. 429.

[5] Catak, S.; Monard, G.; Aviyente, V.; Ruiz-López, M. F. J. Phys. Chem. A 2008, 112, 8752-8761.

[6] Geiger, T.; et al. J. Biol. Chem. 1987, 262, 785-794.

[7] Negussie A. H.; J Control Release. 2010 April 19; 143(2): 265-273.

[8] Enyedi, K. N.; et al. PLoS One 2017, 12, e0178632.

[9] Tripodi A. A. P.; Beilstein J. Org. Chem. 2018, 14, 911-918. 